\title{
Acupuncture's Effects in Treating the Sequelae of Acute and Chronic Spinal Cord Injuries: A Review of Allopathic and Traditional Chinese Medicine Literature
}

\author{
Peter T. Dorsher and Peter M. McIntosh \\ Department of Physical Medicine and Rehabilitation, Mayo Clinic, 4500 San Pablo Road, Jacksonville, Florida 32224, USA \\ Correspondence should be addressed to Peter T. Dorsher, dorsher.peter@mayo.edu
}

Received 27 July 2008; Accepted 12 January 2009

Copyright ( $) 2011$ P. T. Dorsher and P. M. McIntosh. This is an open access article distributed under the Creative Commons Attribution License, which permits unrestricted use, distribution, and reproduction in any medium, provided the original work is properly cited.

\begin{abstract}
Each year, there are an estimated 12000 individuals who sustain a spinal cord injury (SCI) in the United States. Improved understanding of the pathophysiology of SCI and its sequelae has over the past 50 years led to the development of medical treatments (especially urologic) that have enhanced short- and long-term survival from these injuries. The prevalence of individuals with SCI in this country is 250000 individuals; and beyond the incalculable personal consequences of these devastating neurologic injuries, substantial direct and indirect societal costs result from the sequelae of SCI including paralysis, sensory loss, chronic pain, decubiti and bladder and/or bowel incontinence. The purpose of this treatise is to review the allopathic and traditional Chinese medicine (TCM) literature available through MEDLINE, PubMed and eCAM search engines that discuss the potential uses of acupuncture to treat acute and chronic spinal cord injuries and their sequelae, and present the neurophysiologic mechanisms for acupuncture's beneficial effects. There is evidence that use of electroacupuncture in acute SCI may significantly improve long-term neurologic recovery from these injuries both in terms of motor, sensory and bowel/bladder function with essentially no risk. Acupuncture may even improve neurourologic function in individuals with chronic SCI, and help with management with chronic pain associated with these injuries.
\end{abstract}

\section{Introduction}

Due to advances in critical care medicine and spine surgery techniques in the last few decades, more individuals survive severe trauma that result in spinal injuries. The most devastating sequelae of spinal trauma are spinal cord injuries (SCI). The annual incidence of SCI in the United States is $~ 12000 /$ year, with a prevalence of over 250000 persons [1]. Nearly $80 \%$ of individuals sustaining SCI are male; and since 2000, their average age at injury has increased to an average of 39.5 years with $11.5 \%$ of SCI individuals being over the age of 60 [1]. Improved urologic care in the last 50 years has helped prevent the progressive renal failure that previously resulted from recurrent urinary tract infections and hydronephrosis related to bladder dyssynergia and high intravesicular pressures, so that life expectancy of individuals with paraplegia is only about 6 years shorter than their nonSCI peers and only about 10 years shorter for those with low (C5-C8) quadriplegia [1].
Beyond the incalculable personal consequences of SCI, the medical care costs of individuals sustaining SCI are substantial. The lifetime medical costs of a 25-year-old person with high $(\mathrm{C} 1-\mathrm{C} 4)$ quadriplegia is estimated to be over $\$ 3$ million, over $\$ 1.7$ million for those with low quadriplegia, over $\$ 1$ million for paraplegics and over $\$ 680000$ for individuals sustaining incomplete SCI [1]. These figures do not include nearly $\$ 62000 /$ year indirect losses with respect to wages, fringe benefits and productivity [1].

Interventions that improve neurologic recovery from acute SCI would not only potentially improve the quality and quantity of their lives, but would also reduce their health care needs and thus costs. Methyprednisolone use acutely after SCI was the first intervention that appeared to have some influence on the neurologic recovery from SCI [2]. Though statistically significant improvements in motor scores were reported in those receiving methylprednisolone, the clinical significance of these improvements was questionable, and no improvements in myotomal levels were noted [2]. GM-1 
ganglioside [3], 4-aminopyridine [4], nimodipine [5] and naloxone [6] trials likewise failed to improve neurologic outcomes when administered for acute SCI.

The purpose of this study is to review the allopathic and traditional Chinese medicine (TCM) literature examining the use of acupuncture for treating SCIs and their sequelae. Due to significant methodological differences, the TCM reports on acupuncture for treating SCIs will be covered separately. The eCAM, MEDLINE and PUBMED databases were searched with the terms "acupuncture" and "SCI". Reports in English or with an English abstract relevant to the use of acupuncture to treat the sequelae of acute and chronic SCI were reviewed for this study.

\section{Motor and Sensory Recovery}

Less than $1 \%$ of persons with acute SCI experience complete neurologic recovery by hospital discharge [1]. Wong et al. [7] examined the effect of electroacupuncture on neurologic recovery in 100 subjects with acute ASIA A/B traumatic SCIs. Fifty subjects received only standard rehabilitation care, while the other 50 subjects received standard care plus electroacupuncture. Those subjects received $75 \mathrm{~Hz}$ stimulation of surface electrodes at acupuncture points SI-3 (lateral fifth metacarpal) and BL-62 (inferior to lateral malleolus) with auricular acupuncture five times weekly for $30 \mathrm{~min}$ from the time of SCI/surgery to rehabilitation discharge [7]. The cohort receiving electroacupuncture had statistically and clinically significant improvements in their neurologic outcomes at the 1 year mark, compared to those who received standard rehabilitation care [7]. ASIA motor scores were significantly improved in the electroacupuncture group compared to the control group (74.2 versus 52.3 , mean) as were pinprick sensory scores ( 90.0 versus 69.8 , mean), light touch sensory scores ( 92.5 versus 70.5 , mean), and total FIM scores (106.9 versus 88.7 , mean). Of the 50 subjects in the control group receiving only standard rehabilitation care, $32(64 \%)$ remained ASIA A or B 1 year post injury follow up, $8(16 \%)$ improved to ASIA C, six $(12 \%)$ improved to ASIA D and four (8\%) improved to ASIA E [7]. In contrast, the cohort who received electroacupuncture along with standard rehabilitation care showed much better neurologic outcomes. Only 11 (22\%) of the electroacupuncture group remained ASIA A or B 1 year post injury, while $10(20 \%)$ improved to ASIA C, 8 (16\%) improved to ASIA D and 21 (42\%) improved to ASIA E [7]. Seven subjects with ASIA A injuries receiving electroacupuncture improved to ASIA $\mathrm{C} / \mathrm{D} / \mathrm{E}$ at the 1 year mark, compared to none in the control group [7]. Of 22 subjects, 16 with ASIA B injuries receiving electroacupuncture improved to ASIA E at the 1 year follow up, and the other six to ASIA D, while in the control group only 4 of 18 improved to ASIA E status at the 1 year mark and six to ASIA D [7].

Thus, study of Wong et al. suggests a much larger effect of electroacupuncture on ultimate neurologic recovery from acute SCI than any pharmacologic intervention to date. The acupuncture points chosen are described to activate the Governor Vessel meridian, which is described to ascend through the sacrum and spinal column to the base of the skull $[8,9]$. The ankle acupuncture point BL-62 has sensory innervation by the sural nerve, a branch of the sciatic nerve, while the hand acupuncture point SI-3 is innervated by the ulnar nerve. Electrical stimulation at this relatively high frequency $(75 \mathrm{~Hz})$ is known to release enkephalins and dynorphins at the spinal cord level [10], providing a putative endogenous mechanism for limiting the damage to spinal cord neurons and axons following trauma. This landmark study should be replicated by another center(s), and if these impressive results are confirmed, then this treatment should be integrated into the acute management of SCIs.

The study of Wong et al. [7] does not score highly on the Jadad scale, as it does not describe the randomization process for subjects entered in the study, and it is not double-blinded (Table 1). The actual study data, however, shows that the acupuncture and control groups were well matched at study entry in terms of patient ages, duration of SCIs, male/female distributions, quadriplegia/paraplegia proportions, ASIA classifications and FIM scores. The same nurse practitioner did all patient examinations, though the study does not report whether or not the nurse practitioner was blinded to the type of intervention of the subject patients. Double blinding was not an element of the study, as it compared usual rehabilitation care to usual rehabilitation care plus a standardized electroacupuncture intervention.

Electroacupuncture in an animal model of incomplete acute SCI [20] led to more rapid neurologic recovery of proprioception ( $>2$ times more rapidly) and motor function ( $>3$ times more rapidly), though addition of corticosteroid to the electroacupuncture further enhanced the rate of neurologic recovery.

\section{Bladder Dysfunction}

Urinary incontinence related to SCIs has been reported to have a poor long-term prognosis for recovery [21-23]. Prior studies document that up to $41 \%$ of patients with normal neurologic examinations after thoracolumbar spinal injuries demonstrate neurogenic bladders on urodynamic testing [24]. Bulbocavernosus reflex and saddle sensation testing are specific but not sufficiently sensitive to clinically select those patients with neurogenic bladder [24]. A retrospective study by Weiss et al. [25] found that SCI patients without perianal pinprick sensation at initial evaluation never regained volitional voiding, and lack of toe proprioception at initial evaluation strongly predicted failure to recover volitional voiding. A recent case study, however, documented a subject with traumatic SCI who had urinary incontinence with absent saddle sensation at initial presentation who became completely continent of urine and able to void volitionally after treatment with electroacupuncture [26].

Traditionally, treatment of UMN neurogenic bladder incontinence has included behavioral interventions (fluid schedules and regular voiding attempts), bladder catheterization and a variety of medications including anti-cholinergic and alpha blocker agents [23].

There have been a limited number of research studies examining the use of acupuncture to treat neurogenic bladder dysfunction due to UMN SCI. For patients with 
acute SCIs ( $<1$ month), Cheng et al. [13] demonstrated that $20 \mathrm{~Hz}$ electroacupuncture at CV-3, CV-4 and BL-32 helped acute SCI patients with neurogenic bladder achieve balanced (continent, catheter free) bladder function 33\% more rapidly than those receiving only usual care including medications and training in self catheterization. CV-3 and CV-4 are on the midline in the lower abdomen over the location of the bladder and influence parasympathetic nervous system, while BL-32 is at the second sacral foramen. Recall that the sacral plexus at S2-S4 provides parasympathetic innervation to the bladder as well as innervation of the external urinary sphincter via the pudendal nerve. The acupuncture intervention in study of Cheng et al. [13] did not improve bladder function in subjects having complete upper or lower motor neuron SCIs, and it was most efficacious if initiated within 3 weeks of the spinal injuries. That study examined only 60 (out of 80 , total randomized) subjects who ultimately achieved balanced bladder status, and its analysis examined both upper motor and lower motor neuron neurogenic bladders [13]. This study had a Jadad score of 2, as it did not discuss the method of randomization of subjects and was not double blinded, comparing electroacupuncture plus usual bladder retraining to usual bladder training alone (Table 1). Wong et al. [7] demonstrated that electroacupuncture at SI-3 and BL-62 in conjunction with auricular acupoints produced enhanced recovery of bladder function in patients with acute SCI. At 1 year post SCI, subjects receiving the acupuncture intervention demonstrated statistically significant improvements in their bladder functional independence measure (FIM) scores to a mean score of 3.02 compared to subjects in the control group whose mean bladder FIM score was 1.49 [7]. An FIM bladder control score of 3 indicates a subject has no incontinence episodes, and is independent with use of a catheter or uses medication for control, while a score of 1.5 would indicates that the subject has urinary incontinence episodes nearly every day and requires significant physical assistance with bladder management.

Even in subjects with chronic SCI, acupuncture may have a beneficial effect in treating neurogenic bladder dysfunction. Honjo et al. [14] performed manual stimulation of point BL-33 (third sacral foramen) weekly for a month in 13 subjects with chronic SCI, which resulted in resolution of urinary incontinence in $15 \%$ of subjects and at least $50 \%$ improvement in incontinence symptoms in another $46 \%$ [14]. The average bladder volume increased $\sim 2$-fold after four weekly treatments, and was still $70 \%$ improved one month following the last acupuncture treatment [14]. Recall that the parasympathetic outflow to the bladder occurs from the sacral plexus at the S2-S4 spinal levels as does the innervation to the external urethral sphincter via the pudendal nerve. This study had a Jadad score of 1 as it was not randomized and the intervention was not blinded (Table 1).

\section{Neurogenic Bowel}

Incontinence of bowel following SCI can have devastating consequences on re-integration of SCI individuals into work and social situations. The natural history of recovery of bowel function following SCI is not favorable [27]. Traditional treatments of neurogenic bowel dysfunction have included use of stool softeners and fiber to achieve proper stool consistency, then regular use of suppositories or enemas to promote adequate evacuation of the lower colon to minimize risk of gross incontinence or stool leakage.

Wong et al. [7] provide the only study in the literature that provides objective evidence of an intervention which improves the natural history of neurogenic bowel dysfunction following acute SCI, by demonstrating electroacupuncture produced statistically significant $(P=$ .001) improvement in bowel FIM scores 1 year post injury compared to control group receiving no acupuncture (mean score 3.42 versus 1.70 , resp.). A bowel management FIM score of 3 means no incontinence or assistance from another person for bowel care, while a score of 1.7 indicates the need for assistance with bowel care and some episodes of fecal incontinence, though not on a daily basis. Thus, this represents a clinically significant improvement in bowel incontinence.

\section{Pain}

Over $80 \%$ of individuals sustaining SCI experience pain [28]. Approximately $40 \%$ of individuals sustaining traumatic or non-traumatic SCI experience neuropathic pain that in twothirds of cases adversely affects their daily lives, and over $60 \%$ of these individuals report experiencing the pain below the level of the spinal injury [29]. Musculoskeletal pain results from repetitive use of the upper extremities for transfers and wheelchair mobility [30, 31].

Nayak et al. [16] studied 22 patients with SCI who suffered moderate to severe pain of at least six months' duration and received a course of 15 acupuncture treatments over a seven and a half week period after an equivalent assessment period without treatment. Ten patients (46\%) showed improvement in pain intensity and pain sequelae after treatment, whilst six patients (27\%) reported an increase in pain, that was still present 3 months after treatment. The researchers conclude that acupuncture may provide pain relief for at least a subgroup of individuals with SCI and that future research is needed to determine what part of this effect is due to acupuncture [16]. This study had a Jadad score of 1 as it was not randomized and the study intervention was not blinded (Table 1).

Rapson et al. [17], in a retrospective analysis, reported two-thirds of 36 SCI patients with central pain treated with electroacupuncture improved. The type and level of SCI did not predict improvement with acupuncture nor did duration of pain symptoms [17]; but subjects with bilateral, burning pain below the SCI lesion were the most likely to improve $(P=.005)$. This study had a Jadad score of 0 as it was not randomized, the study intervention was not blinded, and was a retrospective clinical series with no discussion of dropouts (Table 1).

Dyson-Hudson et al. [18] studied the effects of acupuncture in 17 wheelchair-using SCI subjects having chronic musculoskeletal shoulder pain. Ten treatments of acupuncture or minimal needling of non-acupuncture points were performed [18], and both groups had statistically significant 
TABLE 1: Prior studies of acupuncture's use in treating SCI sequelae.

\begin{tabular}{|c|c|c|c|c|c|c|}
\hline Study & $\begin{array}{l}\text { Number of } \\
\text { patients }\end{array}$ & Randomized? & $\begin{array}{l}\text { Acupuncture } \\
\text { intervention }\end{array}$ & $\begin{array}{l}\text { Control } \\
\text { intervention }\end{array}$ & $\begin{array}{l}\text { Standardized } \\
\text { acupuncture? }\end{array}$ & Jadad score \\
\hline \multicolumn{7}{|l|}{ All SCI problems } \\
\hline Wong et al. [7] & 100 & Yes & $\begin{array}{l}75 \mathrm{~Hz} \text { surface EA at } \\
\text { SI-3 and BL-62 } \\
\text { plus auricular } \\
\text { acupoints plus } \\
\text { usual care }\end{array}$ & $\begin{array}{l}\text { Usual SCI } \\
\text { rehabilitation } \\
\text { care }\end{array}$ & Yes & 2 \\
\hline Wang [11] & Not given & No & Wide variety & Not applicable & No & $\begin{array}{l}0, \text { retrospective } \\
\text { experience }\end{array}$ \\
\hline Gao et al. [12] & 261 & No & Wide variety & Not applicable & No & $\begin{array}{l}0 \text {, retrospective } \\
\text { experience }\end{array}$ \\
\hline \multicolumn{7}{|l|}{ SCI bladder problems } \\
\hline Cheng et al. [13] & 60 & Yes & $\begin{array}{l}\text { EA } 20-30 \mathrm{~Hz} \text { at } \\
\mathrm{CV}-3, \mathrm{CV}-4 \text {, and } \\
\text { bilateral BL-32 } \\
\text { plus usual care }\end{array}$ & Usual care & Yes & 2 \\
\hline Honjo et al. [14] & 13 & No & $\begin{array}{l}\text { A manual } \\
\text { stimulation at } \\
\text { BL-33 }\end{array}$ & Not applicable & Yes & 1, no dropouts \\
\hline Zhou et al. [15] & 84 & Yes & $\begin{array}{l}\text { EA at Baliao and } \\
\text { BL-35 }\end{array}$ & $\begin{array}{l}\text { EA at } \\
\text { "acupoints } \\
\text { routinely } \\
\text { selected" }\end{array}$ & Yes & $\begin{array}{l}\text { Insufficient } \\
\text { information }\end{array}$ \\
\hline \multicolumn{7}{|l|}{ Pain problems } \\
\hline Nayak et al. [16] & 20 & No & A, no stimulation & Not applicable & $\begin{array}{l}\text { No, some } \\
\text { points } \\
\text { standardized }\end{array}$ & $\begin{array}{l}\text { 1, efficacy study } \\
\text { with } 2 \text { dropouts }\end{array}$ \\
\hline Rapson [17] & 36 & No & EA & Not applicable & Yes & $\begin{array}{l}0, \text { retrospective } \\
\text { experience }\end{array}$ \\
\hline Dyson-Hudson et al. [18] & 17 & Yes & $\begin{array}{l}\text { A, manual } \\
\text { stimulation }\end{array}$ & $\begin{array}{l}\text { Sham superficial } \\
\text { acupuncture }\end{array}$ & $\begin{array}{l}\text { No, but } \\
\text { selected from } \\
\text { a group of } \\
\text { points }\end{array}$ & 5 \\
\hline \multicolumn{7}{|l|}{ Dyreflexia problems } \\
\hline Averill et al. [19] & 15 & No & A, no stimulation & Not applicable & $\begin{array}{l}\text { Yes, "specific } \\
\text { points above } \\
\text { and below } \\
\text { the lesion" }\end{array}$ & $\begin{array}{l}\text { 1, "acupuncture } \\
\text { analgesia study" } \\
\text { no dropouts }\end{array}$ \\
\hline
\end{tabular}

Acupuncture (A) or electroacupuncture (EA).

reductions in shoulder pain decreased significantly with treatment (66\% and $43 \%$ reduction, resp.). There was no statistically significant difference between the two groups (though study power was low), but there was a medium effect size associated with the acupuncture treatment [18]. This study had a Jadad score of 5, reflecting the investigators' adherence to CONSORT guidelines for reporting clinical trials (Table 1).

Acupuncture is known to release endogenous opioids including endorphins, and its pain relieving effects can be blocked by naloxone $[32,33]$. Overall, acupuncture may be a useful adjunct to treat neuropathic and/or musculoskeletal pain following SCI.

\section{Autonomic Hyper-Reflexia}

Though no studies have looked at the use of acupuncture to treat autonomic dysreflexia associated with SCI that can occur with cord lesions at or above T8, Averill et al. [19] studied the effects of acupuncture needle insertion above and below the spinal injury level of 15 patients with SCI who were at risk for autonomic dysreflexia. Though blood pressures overall remained stable over 15 treatment sessions, three individuals did have acute systolic blood pressure elevations (>20 mmHg or higher) that suggested incipient dysreflexia. The authors concluded that blood pressure should be monitored in at risk SCI patients receiving acupuncture [19]. 
This study had a Jadad score of 1 as it was not randomized and the study intervention was not blinded (Table 1).

A recent study by Flachskampf et al. [34] provided the first rigorous, randomized trial of the use of acupuncture to treat hypertension. This study's results suggest that acupuncture produces blood-pressure reductions comparable to those produced by ACE-inhibitor monotherapy or aggressive lifestyle changes, including radical salt restrictions, in mild to moderate hypertension. Acupuncture's beneficial effects dissipated after 3 months, however, indicating the need for ongoing treatments to maintain blood-pressure reductions [34]. The degree of blood-pressure reductions from this intervention were too small to be of clinical utility in autonomic dysreflexia, but other acupuncture treatment protocols such as needling the sympathetic switches [35] should be studied to determine if acupuncture could help treat the blood-pressure elevation associated with autonomic hyper-reflexia non-pharmacologically.

\section{Decubiti}

There are no experimental acupuncture studies that specifically examine its effect on decubiti, though Wang [11] reported that direct current stimulation with $3 \mathrm{~V}$ battery across a saline soaked gauze pad has "long been used ... to treat paraplegic bed sores". Electrical stimulation around the site of decubiti has been demonstrated to enhance the rate of wound healing $[36,37]$. Thus, electroacupuncture around a decubiti would be expected to enhance the rate of decubitus wound healing, and should be the subject of a future study.

\section{Sexual Dysfunction}

There are no acupuncture studies specifically examining its effect on sexual functioning after SCI. Wang [11] reported that sexual dysfunction related to SCI can be "cured" with electroacupuncture in $92.5 \%$ of those individuals, though no supporting evidence was provided in that report.

\section{Traditional Chinese Medicine Literature on Acupuncture for SCI}

There are two main reports most frequently cited regarding the use of TCM to treat SCI and their sequelae. These are the retrospective analyses by Wang [11] in 1992 and Gao et al. [12] in 1996. These are reported here as a separate section since both these studies reflect the methodological differences present throughout the TCM literature. Literature in this time interval by TCM practitioners in Asia reflects acupuncture's accepted efficacy there; so clinical studies usually do not include sham treatment arms which would be considered unethical.

Wang [11] reported a survey of TCM treatment of traumatic paraplegia which the paper defined as "sensory disturbance in the lower limbs and the accompanying loss of motor function along with urinary and defecation dysfunction." The paper actually discusses acupuncture point selection for treating the sequelae of quadriplegia and paraplegia, as well as upper and lower motor neuron injuries. The patient cohort is not fully described, and also appears to include individuals with complete and incomplete SCI. Electroacupuncture performed 12 times per week for 3 months was stated to have $100 \%$ "effectiveness" in treating urinary and fecal disturbances due to SCI, but no description of what "effectiveness" means [11]. No urodynamic confirmation of neurogenic bladder dysfunction is provided before or after acupuncture intervention. This survey concluded that "electric needling of the spinal cord ... is the treatment of choice ... by its marked therapeutic effect on the recovery and re-establishment of motor, urinary bladder, and sexual functions, with a total cure rate of 92.5\%" [11]. The discussion section of this report, however, also intimates that complete SCI does not respond to acupuncture intervention [11].

Gao et al. [12] reported their retrospective analysis of 261 persons with "complete traumatic paraplegia"; but similar to the report of Wang [11], the patient descriptions appear to include those with quadriplegia and paraplegia as well as upper and motor lower neuron weakness. It is not clear from this report's descriptions that the subjects had complete injuries as saddle sensation was not reported and their subjects had "loss of flexor muscle ability to extend" [12]. Fifty six of the subjects had duration of SCI $<1$ year, and 24 of those $<6$ months. Of the eight subjects who were "essentially cured" by acupuncture with the ability to "walk freely without any sort of help ... almost voluntary urination" [12], their injuries were all $<6$ months duration and thus may instead reflect spontaneous neurologic improvement of their injuries. 35\% of subjects were reported to have experienced "marked effectiveness" from treatment defined as "partial recovery of the functions of the nervous system" with ability to "walk on crutches" and "restoration of bladder reflex"; and 57\% were "improved" defined as "some improvement of the functions of the nervous system, including movement and defecation and/or urination" [12]. The lack of adequate descriptions of the subjects and objective data/measurements of their function makes interpretation of these results impossible.

One study from the TCM literature with an English abstract available by Zhou et al. [15] examined the effect of electroacupuncture on urinary retention due to SCI. Out of 84 study subjects, 46 had received electroacupuncture, which produced a positive effect on their urinary retention in $82.6 \%$ (versus $63.2 \%$ in control subjects), and a urinary retention "cure" rate of $43.5 \%$ (versus $23.7 \%$ in controls). The abstract did not delineate the acuity of the SCI, their types/levels, or other details of the subjects studied or interventions provided.

Another TCM study with an English abstract by Yu [38] reported that transcutaneous electrical stimulation at acupoints could reduce spasticity related to SCI. High-frequency electrical stimulation $(100 \mathrm{~Hz})$ produced an immediate antispastic effect, but required ongoing treatment, which was postulated to enhance the production of dynorphin in the anterior horn of the spinal cord that decreases the excitability of the motor neurons via kappa opiate receptor activation. These findings were confirmed by the study of Dong et al. 


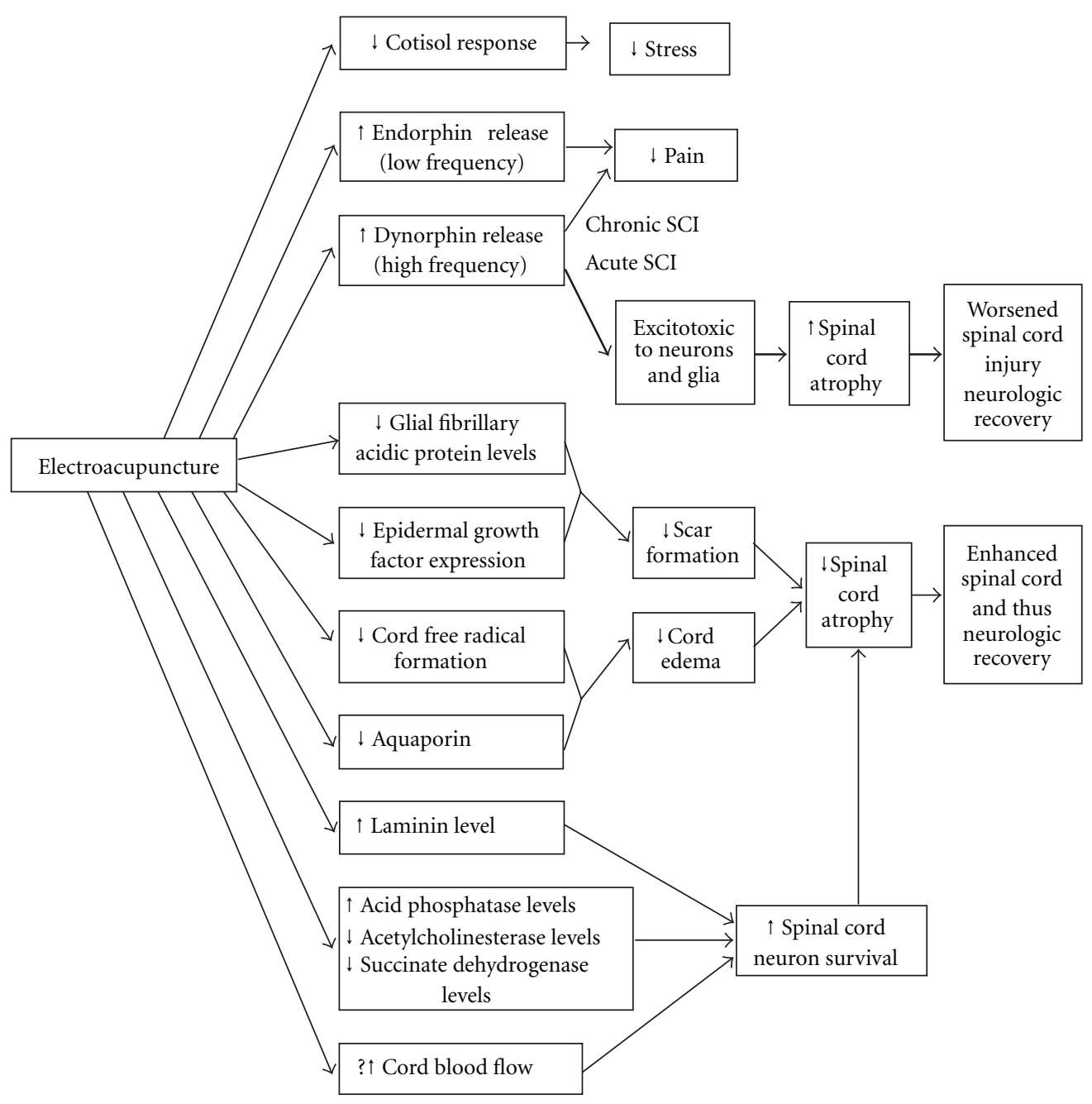

FIgUre 1: Possible mechanisms of acupuncture's effects in treating SCIs.

[39] using $100 \mathrm{~Hz}$ stimulation to reduce spasticity in a rat acute SCI model.

\section{Possible Mechanisms for Acupuncture's Effects}

The potential mechanisms for acupuncture's beneficial clinical effects in treating the sequelae of SCI acutely are shown in Figure 1. In animal experimental models of acute SCI, electroacupuncture has been demonstrated to have a number of positive physiologic effects at the site of cord injury. Electroacupuncture produces reduced glial fibrillary acidic protein levels in the injured cord $[40,41]$, which serves to inhibit reactive astrocyte proliferation and reduce glial scar formation. Electroacupuncture also produces reduced epidermal growth factor receptor levels [41], also suggesting less scar formation. Electroacupuncture also reduces free radical formation [42], and down-regulates AQP-4 (aquaporin) expression after SCI [43] so as to inhibit spinal cord edema that can produce secondary spinal cord damage. Electoacupuncture also reduces spinal cord atrophy due to
SCI with two-thirds reduction of anterior horn neuron loss [44] in acute SCI models in animals, and reduces the acute stress response of those animal as measured by the serum cortisol levels.

Enhanced spinal cord regeneration potential of electroacupuncture is implied by the findings of earlier and higher levels of laminin expression in the injured cord in animals treated with electroacupuncture [45], and by elevated levels of acid phosphatase during the recovery period [46, 47]. Wu et al. [47] reported that acupuncture reverses the elevations of acetylcholinesterase and succinate dehydrogenase and reductions of acid phosphatase seen in the anterior horn of the spinal cord involved by experimental SCI, which could inhibit or delay the deterioration (and possibly promote the recovery) of those anterior horn cell neurons.

Uchida and Hotta [48] have demonstrated acupuncturelike stimuli can increase cortical and uterine blood flow, and may provide another mechanism for acupuncture's effects in limiting damage and enhancing recovery of acute SCI. Electroacupuncture at low frequencies $(\sim 2 \mathrm{~Hz})$ produces endorphin release by the central nervous system [49] whose 
effect can be blocked by naloxone, while high frequency electroacupuncture causes release of dynorphins [10]. The effects of dynorphins on spinal cord neurons and glia are complex, as dynorphins may have neuroprotective or proapoptotic actions on spinal cord cells, depending on the distribution of kappa opioid receptors and the amount of dynorphin released [50]. Excessive dynorphin levels in acute SCI likely contribute to hyperalgesia as well as excitotoxic injury to neurons and glia, while in chronic SCI dynorphins released at normal physiologic concentrations through electroacupuncture may have analgesic and neuroprotective effects on spinal cord cells [50].

\section{Conclusions}

There is evidence that use of electroacupuncture in acute spinal cord injured subjects may significantly improve their long-term neurologic recovery including motor, sensory and bowel/bladder function. Acupuncture may even improve neurourologic function in spinal cord injured individuals with chronic neurogenic bladder, and may also be a useful adjunct in the management of their chronic neuropathic and musculoskeletal pain conditions. The acupuncture effects appear to result from stimulation of appropriate spinal cord segmental levels or peripheral nerves, and the known release of endogenous opioids at the spinal cord level produced by acupuncture treatment and electrical stimulation of peripheral nerves provides a plausible mechanism for its effects in pain relief and limiting SCI after acute trauma. At least theoretically, high frequency electroacupuncture $(>100 \mathrm{~Hz})$ in acute SCI may worsen excessive local dynorphin release that is excitotoxic to neurons and glial cells.

The acupuncture literature on its use for treating the sequelae of acute and chronic SCI in humans is limited, and demonstrates wide variations in methodology both in terms of use of control interventions and types of acupuncture interventions. More recent studies, in general, are of higher methodologic quality and more closely adhere to the CONSORT criteria. Older studies, prior to 2000, tend to be more anecdotal, retrospective descriptions of the results of extensive clinical experience using acupuncture to treat the sequelae of human SCI. Though those retrospective studies do not provide the detailed patient or methodologic data that would permit rigorous scientific conclusions of acupuncture's effects in treating the sequelae of acute and chronic SCIs, they do provide important guidance on TCM concepts and point selections recommended by experts for treating the sequelae of SCI.

\section{References}

[1] "Spinal Cord Injury Information Network," January 2008, http://www.spinalcord. uab.edu/show.asp?durki=21446.

[2] M. B. Bracken, M. J. Shepard, T. R. Holford et al., "Administration of methylprednisolone for 24 or 48 hours or tirilazad mesylate for 48 hours in the treatment of acute spinal cord injury: results of the Third National Acute Spinal Cord Injury randomized controlled trial," Journal of the American Medical Association, vol. 277, no. 20, pp. 1597-1604, 1997.
[3] P. Chinnock and I. Roberts, "Gangliosides for acute spinal cord injury," Cochrane Database of Systematic Reviews, no. 2, article, Article ID CD004444, 2005.

[4] D. DeForge, J. Nymark, E. Lemaire et al., "Effect of 4aminopyridine on gait in ambulatory spinal cord injuries: a double-blind, placebo-controlled, crossover trial," Spinal Cord, vol. 42, no. 12, pp. 674-685, 2004.

[5] M. E. Petitjean, V. Pointillart, F. Dixmerias et al., "Medical treatment of spinal cord injury in the acute stage," Annales Françaises d'Anesthésie et de Réanimation, vol. 17, pp. 114-122, 1998.

[6] M. B. Bracken, M. J. Shepard, W. F. Collins et al., "A randomized, controlled trial of methylprednisolone or naloxone in the treatment of acute spinal-cord injury. Results of the Second National Acute Spinal Cord Injury Study," The New England Journal of Medicine, vol. 322, no. 20, pp. 1405-1411, 1990.

[7] A. M. K. Wong, C.-P. Leong, T.-Y. Su, S.-W. Yu, W.-C. Tsai, and C. P. C. Chen, "Clinical trial of acupuncture for patients with spinal cord injuries," American Journal of Physical Medicine and Rehabilitation, vol. 82, no. 1, pp. 21-27, 2003.

[8] P. Deadman, M. Al-Khafaji, and K. Baker, A Manual of Acupuncture, Hove, Journal of Chinese Medicine Publications, East Sussex, UK, 1998.

[9] G. Maciocia, The Channels of Acupuncture, Elsevier, Philadelphia, Pa, USA, 2006.

[10] J.-S. Han, "Acupuncture and endorphins," Neuroscience Letters, vol. 361, no. 1-3, pp. 258-261, 2004.

[11] H. Wang, "A survey of the treatment of traumatic paraplegia by traditional Chinese medicine," Journal of Traditional Chinese Medicine, vol. 12, no. 4, pp. 296-303, 1992.

[12] X. Gao, C. Gao, J. Gao et al., "Acupuncture treatment of complete traumatic paraplegia-analysis of 261 cases," Journal of Traditional Chinese Medicine, vol. 16, no. 2, pp. 134-137, 1996.

[13] P.-T. Cheng, M.-K. Wong, and P.-L. Chang, "A therapeutic trial of acupuncture in neurogenic bladder of spinal cord injured patients-a preliminary report," Spinal Cord, vol. 36, no. 7, pp. 476-480, 1998.

[14] H. Honjo, Y. Naya, O. Ukimura, M. Kojima, and T. Miki, "Acupuncture on clinical symptoms and urodynamic measurements in spinal-cord-injured patients with detrusor hyperreflexia," Urologia Internationalis, vol. 65, no. 4, pp. 190195, 2000.

[15] L. Y. Zhou, J. Li, C. M. Li et al., "Observation on therapeutic effect of electroacupuncture at points Baliao and Huiyang (BL 35) on retention of urine induced by spinal cord injury," Zhongguo Zhen Jiu, vol. 26, no. 4, pp. 237-239, 2006.

[16] S. Nayak, S. C. Shiflett, N. E. Schoenberger et al., "Is acupuncture effective in treating chronic pain after spinal cord injury?" Archives of Physical Medicine and Rehabilitation, vol. 82, no. 11, pp. 1578-1586, 2001.

[17] L. M. Rapson, N. Wells, J. Pepper, N. Majid, and H. Boon, "Acupuncture as a promising treatment for below-level central neuropathic pain: a retrospective study," Journal of Spinal Cord Medicine, vol. 26, no. 1, pp. 21-26, 2003.

[18] T. A. Dyson-Hudson, P. Kadar, M. LaFountaine et al., "Acupuncture for chronic shoulder pain in persons with spinal cord injury: a small-scale clinical trial," Archives of Physical Medicine and Rehabilitation, vol. 88, no. 10, pp. 1276-1283, 2007. 
[19] A. Averill, A. C. Cotter, S. Nayak, R. J. Matheis, and S. C. Shiflett, "Blood pressure response to acupuncture in a population at risk for autonomic dysreflexia," Archives of Physical Medicine and Rehabilitation, vol. 81, no. 11, pp. 14941497, 2000.

[20] J. W. Yang, S. M. Jeong, K. M. Seo, and T. C. Nam, "Effects of corticosteroid and electroacupuncture on experimental spinal cord injury in dogs," Journal of Veterinary Science, vol. 4, no. 1, pp. 97-101, 2003.

[21] D. Chou, R. Hartl, and V. K. H. Sonntag, "Conus medullaris syndrome without lower-extremity involvement in L-1 burst fractures: report of four cases," Journal of Neurosurgery: Spine, vol. 4, no. 3, pp. 265-269, 2006.

[22] P. J. Shenot, D. A. Rivas, T. Watanabe, and M. B. Chancellor, "Early predictors of bladder recovery and urodynamics after spinal cord injury," Neurourology and Urodynamics, vol. 17, no. 1, pp. 25-29, 1998.

[23] H. Madersbacher, "Neurogenic bladder dysfunction," Current Opinion in Urology, vol. 9, pp. 303-307, 1999.

[24] T. Watanabe, A. R. Vaccaro, H. Kumon, W. C. Welch, D. A. Rivas, and M. B. Chancellor, "High incidence of occult neurogenic bladder dysfunction in neurologically intact patients with thoracolumbar spinal injuries," Journal of Urology, vol. 159, no. 3, pp. 965-968, 1998.

[25] D. J. Weiss, G. W. Fried, M. B. Chancellor, G. J. Herbison, J. F. Ditunno Jr., and W. E. Staas Jr., "Spinal cord injury and bladder recovery," Archives of Physical Medicine and Rehabilitation, vol. 77, no. 11, pp. 1133-1135, 1996.

[26] P. T. Dorsher, R. Reimer, and E. Nottmeier, "Treatment of urinary incontinence due to incomplete spinal cord injury with acupuncture and percutaneous electrical nerve stimulation: two cases and literature review," Medical Acupuncture, vol. 21, no. 1, pp. 21-26, 2009.

[27] M. C. Schönherr, J. W. Groothoff, G. A. Mulder, and W. H. Eisma, "Functional outcome of patients with spinal cord injury: rehabilitation outcome study," Clinical Rehabilitation, vol. 13, no. 6, pp. 457-463, 1999.

[28] D. D. Cardenas and M. P. Jensen, "Treatments for chronic pain in persons with spinal cord injury: a survey study," Journal of Spinal Cord Medicine, vol. 29, no. 2, pp. 109-117, 2006.

[29] L. Wershagen, Analysis of neuropathic pain after spinal cord injury, Doctoral thesis, Karolinska Intsitutet, 2008.

[30] E.G. Widerstrom-Noga, E. Felipe-Cuervo, and R. P. Yezierski, "Chronic pain after spinal injury:interference with sleep and daily activities," Archives of Physical Medicine and Rehabilitation, vol. 82, no. 11, pp. 1571-1577, 2001.

[31] W. P. Waring and F. M. Maynard, "Shoulder pain in acute traumatic quadriplegia," Paraplegia, vol. 29, no. 1, pp. 37-42, 1991.

[32] B. Pomeranz, "Do endorphins mediate acupuncture analgesia?" Advances in Biochemical Psychopharmacology, vol. 18, pp. 351-359, 1978.

[33] J. Shen, "Research on the neurophysiological mechanisms of acupuncture: review of selected studies and methodological issues," Journal of Alternative and Complementary Medicine, vol. 7, no. 1, pp. S121-S127, 2001.

[34] F. A. Flachskampf, J. Gallasch, O. Gefeller et al., "Randomized trial of acupuncture to lower blood pressure," Circulation, vol. 115, no. 24, pp. 3121-3129, 2007.

[35] J. Y. Wong, A Manual of Neuroanatomic Acupuncture: Volume 2: Neurologic Disorders, Pain and Stress Clinic, Toronto, Canada, 2001.
[36] J. M. Wood, P. E. Evans III, K. U. Schallreuter et al., "A multicenter study on the use of pulsed low-intensity direct current for healing chronic stage II and stage III decubitus ulcers," Archives of Dermatology, vol. 129, no. 8, pp. 999-1009, 1993.

[37] M. C. Biedebach, "Prevention and treatment of pressure ulcers using electrical stimulation," in Pressure Ulcer Research: Current and Future Perspectives, D. Bader, C. Bouten, D. Colin, and C. Domens, Eds., pp. 89-107, Springer, Berlin, Germany, 2005.

[38] Y. Yu, "Transcutaneous electric stimulation at acupoints in the treatment of spinal spasticity: effects and mechanism," Chinese Medical Journal, vol. 73, no. 10, pp. 593-595, 1993, 637.

[39] H.-W. Dong, L.-H. Wang, M. Zhang, and J.-S. Han, "Decreased dynorphin a (1-17) in the spinal cord of spastic rats after the compressive injury," Brain Research Bulletin, vol. 67, no. 3, pp. 189-195, 2005.

[40] C. Yang, B. Li, T. S. Liu, D. M. Zhao, and F. A. Hu, "Effect of electroacupuncture on proliferation of astrocytes after spinal cord injury," Zhongguo Zhen Jiu, vol. 25, pp. 569-572, 2005.

[41] B. Peng, X. F. Meng, M. Li et al., "Effects of electroacupuncture on the expression of epidermal growth factor receptor and glial fibrillary acidic protein after spinal cord injury in rats," Zhen Ci Yan Jiu, vol. 32, no. 4, pp. 219-223, 2007.

[42] Y. Wu, Z. Sun, Z. Li, Y. Zhao, and S. Sun, "Effect of acupuncture on free radicals in rats with early experimental spinal cord injury," Journal of Traditional Chinese Medicine, vol. 22, no. 1, pp. 51-54, 2002.

[43] Q. M. Han, J. Xie, S. T. Chai, J. Fang, and Q. Liu, "Effect of Governer Meridian electro-acupuncture on water channel aquaporin-4 in experimental spinal cord injured rats," Zhongguo Zhong Yi Yan Jiu Yuan Zhu Ban, vol. 25, no. 7, pp. 637-639, 2005.

[44] M. J. Politis and M. A. Korchinski, "Beneficial effects of acupuncture treatment following experimental spinal cord injury: a behavioral morphological, and biochemical study," Acupuncture and Electrotherapeutics Research, vol. 15, no. 1, pp. 37-49, 1990.

[45] Z. Zhu, "Effects of electroacupuncture on laminin expression after spinal cord injury in rats," Zhongguo Zhong Xi Yi Jie He Za Zhi, vol. 22, no. 7, pp. 525-527, 2002.

[46] Z. Jin, Z. Tao, W. Ren, and X. Du, "Electro-acupuncture effects on experimental spinal cord injury of the cat as evaluated by acid phosphatase detection," Acupuncture Research, vol. 21, no. 4, pp. 50-53, 1996.

[47] Y. Wu, C. Liu, and Q. Chen, "Effect of acupuncture on enzymology of motor neuron of anterior horn of experimental spinal cord injury in rats," Zhongguo Zhong Yi Yan Jiu Yuan Zhu Ban, vol. 19, no. 12, pp. 740-742, 1999.

[48] S. Uchida and H. Hotta, "Acupuncture affects regional blood flow in various organs," Evidence-Based Complementary and Alternative Medicine, vol. 5, no. 2, pp. 145-151, 2008.

[49] S. X. Ma, "Neurobiology of acupuncture: toward CAM," Evidence-Based Complementary and Alternative Medicine, vol. 1, pp. 41-47, 2004.

[50] K. F. Hauser, J. V. Aldrich, K. J. Anderson et al., "Pathobiology of dynorphins in trauma and disease," Frontiers in Bioscience, vol. 10, no. 1, pp. 216-235, 2005. 


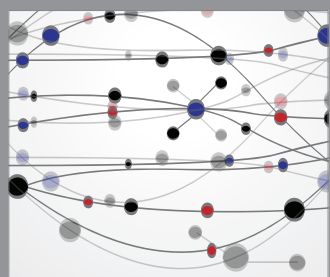

The Scientific World Journal
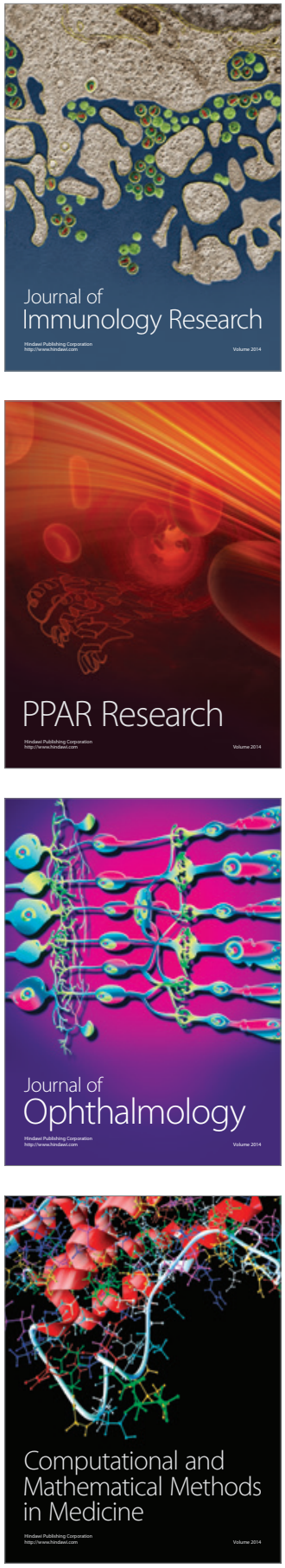

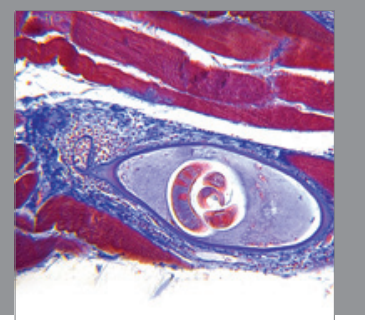

Gastroenterology

Research and Practice
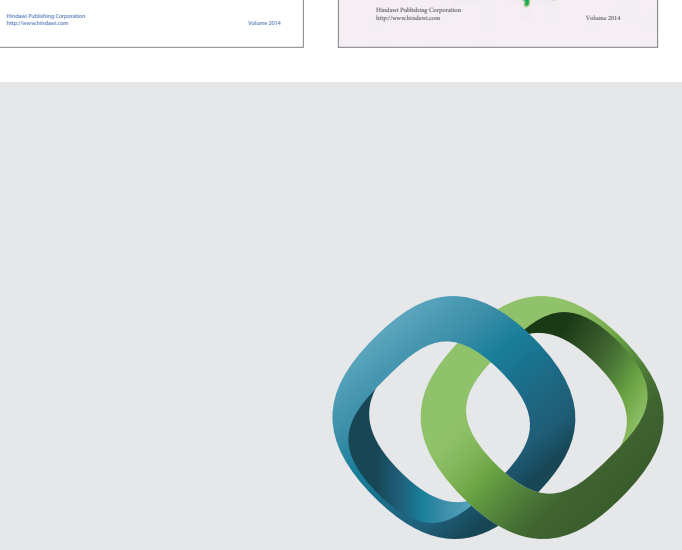

\section{Hindawi}

Submit your manuscripts at

http://www.hindawi.com
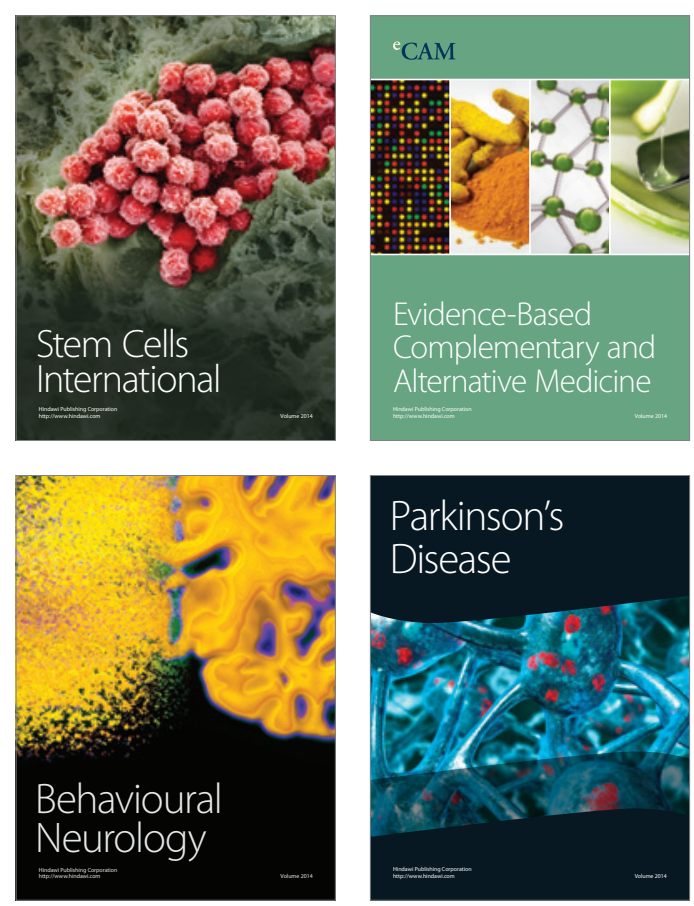

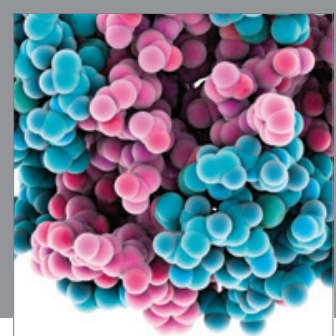

Journal of
Diabetes Research

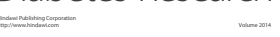

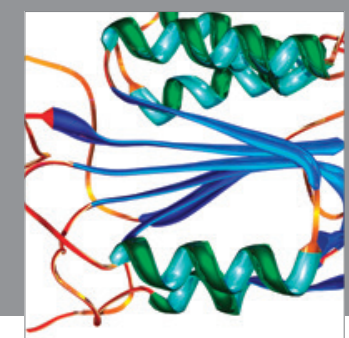

Disease Markers
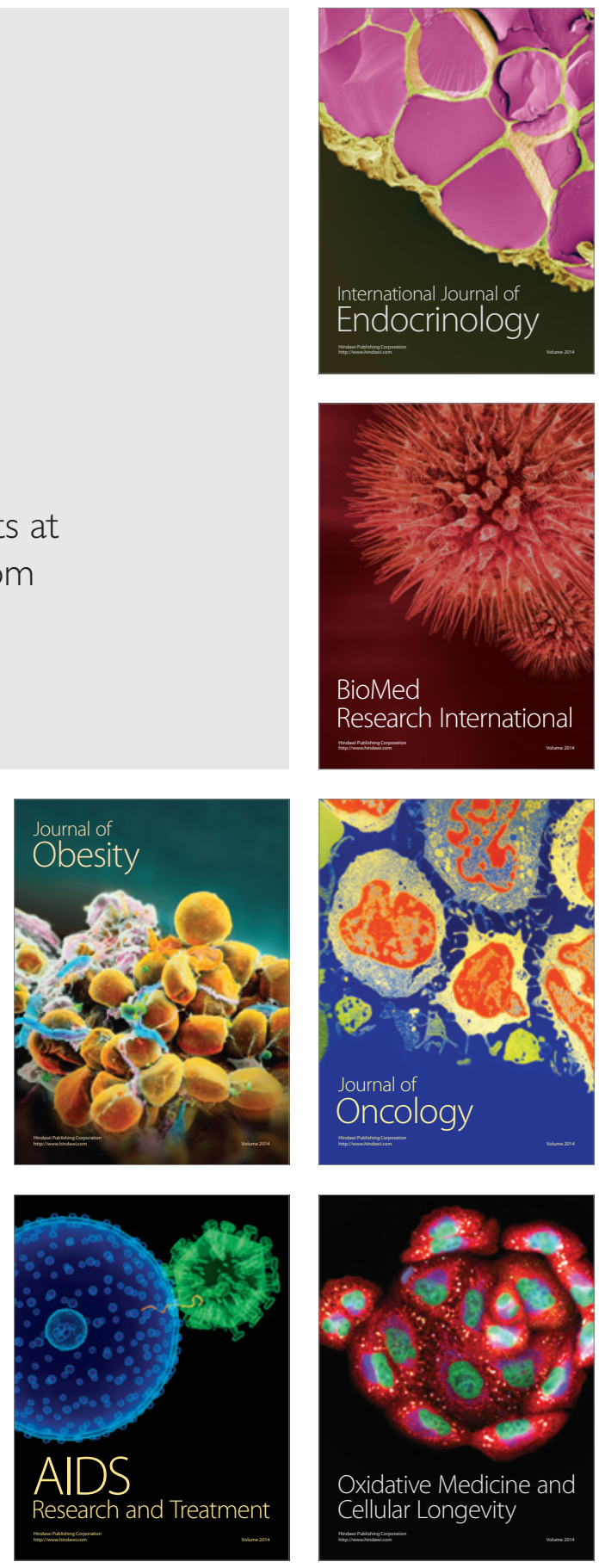